\title{
ADVANCED MAGNETIC MATERIALS FOR AERONAUTICS
}

\author{
Miroslav Šmelko \\ Dušan Praslička \\ Josef Blažek \\ Technical University of Košice, Faculty of Aeronautics, \\ Košice, Slovakia
}

\begin{abstract}
In the field of magnetic sensors, magnetic microwires with positive magnetostriction are the materials of the future. Their mechanical and magnetic properties render them ideal materials for applications in aeronautics. A single microwire with a $40 \mu \mathrm{m}$ diameter and a length of $10 \mathrm{~mm}$ is capable of capturing information about tensile stresses, magnetic fields, temperature and distance. This information is carried by a parameter called the Switching Field, $H_{S W}$, which is specific for different types of microwire. Numerous physical qualities affect the $H_{S W}$ and through sensing of $H_{S W}$, these qualities may be quantified. (A number of physical qualities affecting $H_{S W}$ can be sensed and quantified by means of a contactless induction method.) What distinguishes the system developed by the present authors from other measuring systems based on magnetic microwires is the positioning of a microwire outside the coil system. Thanks to this improvement it is possible to use microwires embedded directly in the construction material. Small dimensions microwires do not damage the structure of the construction material. The absence of a galvanic connection makes this technology even more interesting compared with traditional forge gauges. Offering the possibility of the simultaneous measuring of four parameters, this technology can be used in a wide range of aviation applications. Measurements of an external magnetic field can be used for the navigation and stabilization of an aerial vehicle. Tensile stress and distance measuring can be helpful to understand some processes occurring under the surface of the construction material and also to perform fatigue monitoring or structure load monitoring. Another big advantage of magnetic microwires is the low price. Just 1 gram of base material is sufficient to prepare about $40 \mathrm{~km}$ of microwire. All these features combine to offer us a material ideal for Smart Sensors, possibly available for use in the near future.
\end{abstract}

Keywords: Microwires, Strain Gauge, Mechanical Stress, Contactless

\section{INTRODUCTION}

Composite materials are frequently used in a wide range of applications. Composite structures allow embedding micro-scale sensors during the production phase. If sensor dimensions are as small as the material matrix, sensors do not damage the composite. Magnetic microwires satisfy this requirement. While the diameter of the composite matrix glass fiber ranges between 3 to 13 $\mu \mathrm{m}$, a diameter of microwire is anywhere between $100 \mathrm{~nm}$ and $100 \mu \mathrm{m}$. The microwire diameter can be set with respect to a concrete composite matrix and can be embedded in the final composite without damaging/adversely affecting the properties of the material.

Magnetic microwires are composite materials with a metal core and a glass coating. Their magnetic properties are determined by the chemical composition of the metal core, core-to-cover 
diameter ratio, production processing and post-processing. The present work shows that magnetic microwires are dependable on a number of physical quantities such as an external magnetic field, tensile stress and temperature. Information about the microwire status is carried by a parameter called Switching Field, $\mathrm{H}_{\mathrm{Sw}}$. Amorphous microwires with high positive magnetostriction are characterized by a rectangular hysteresis loop.

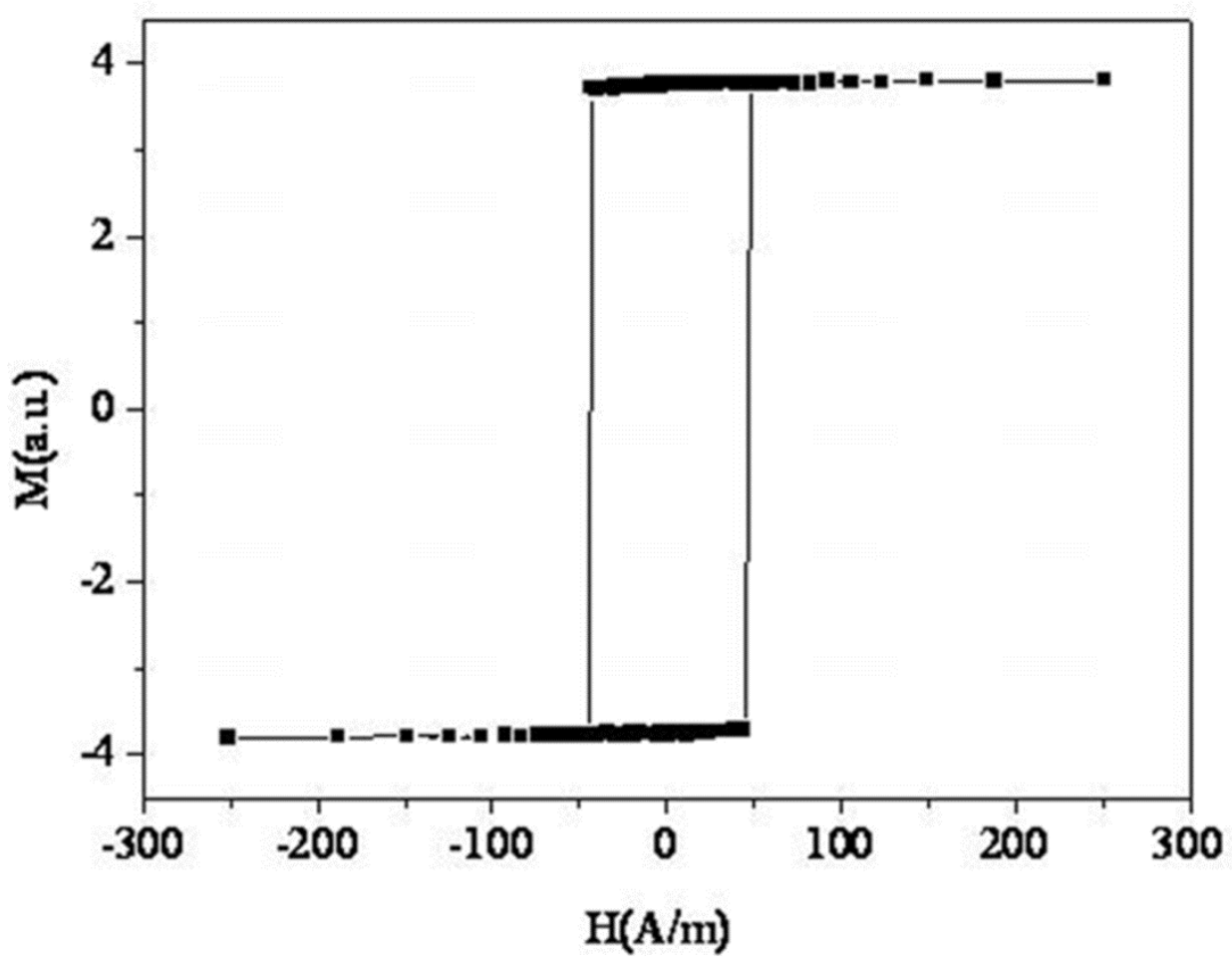

Fig. 1. Rectangular hysteresis loop

The domain structure of such microwire consists of one axial domain in the centre of the wire, covered by radial domains. Such a domain structure exhibits bistable behavior, see Fig.1. Magnetization of the microwire has only two states: positive and negative saturation $\left(\mathrm{M}_{\mathrm{S}+}\right.$ and $\mathrm{M}_{\mathrm{S}-\text { ). }}$ Changing between these two states runs through a single Barkhausen jump, when the external magnetic field reaches the value of the microwire switching field $\mathrm{H}_{\mathrm{Sw}}$. Magnetic properties of microwires are determined by the megnetoelastic interaction betweenthe magnetic moment and the mechanical stresses, thus the switching field is very sensitive to the applied mechanical stress [1]. Due to different thermal expansion coefficients of the metal core and the glass coating, additional stresses are applied to the metal core, hence the switching field is sensitive to temperature, too.

\section{MEASURING METHOD}

An induction method is frequently used for the determination of the switching field [2] [3] [4]. We used the excitation coil fed by the precision triangular shape current, and the pick-up coil for the detection of the motion of the domain wall, represented as induced voltage peaks (see Fig.2). 


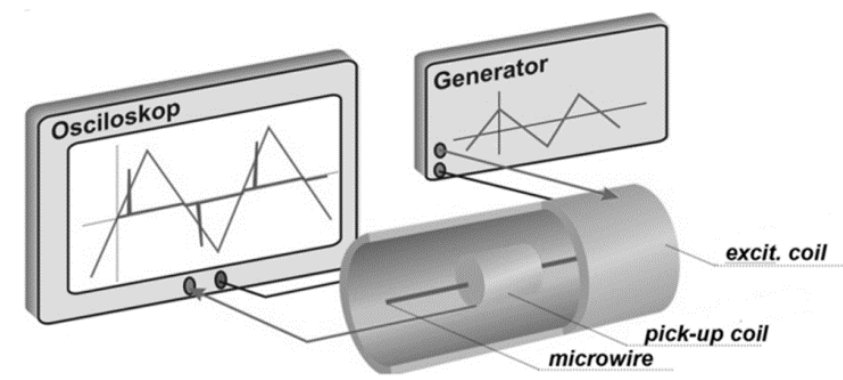

Fig. 2. Induction method of switching field HSW measurement

An excitation field was created by the flat coil with 220 turns while the sensing coil was of the race-track type with 50 turns. The coils were attached to the surface of the sample.

While the exciting field has a triangular shape, the switching field is proportional to the time $t$ in which the peak appears (see Fig. 3). The time shifts between the excitation magnetic field and the induced voltage peaks allow for the determination of the switching field.

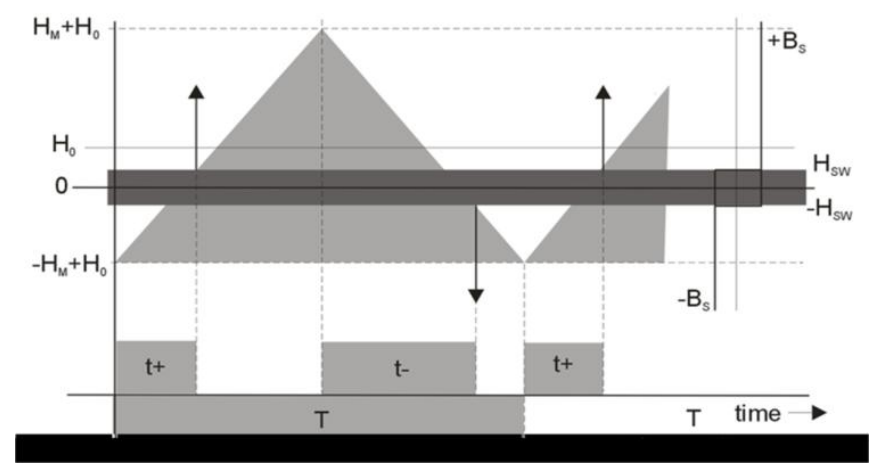

Fig. 3. The measurement of the switching field

The switching field could be then determined by measuring times $t_{+}$and $t_{-}$and can be expressed as follows [2]:

$$
H_{S W}=\frac{2 H_{M}}{T}\left(t_{+}+t_{-}\right)-H_{M}
$$

where $\mathrm{H}_{M}$ is the peak field of the triangular excitation field, $\mathrm{T}$ is the period of the excitation field and $t_{+}$and $t_{-}$are time shifts between the excitation field and the induced voltage peaks.

The formula clearly shows that the switching field could be easily determined by measuring time intervals in direct digital form without affecting the external field $\left(\mathrm{H}_{0}\right)$. Applied stress is then trace ably proportional to the switching field, which is lineally proportional to the microwire response given by the equation (1).

$$
\sigma_{a} \approx H_{s w} \approx t_{+}+t_{-}
$$

The CPLD (complex programmable logical device) controlled electronics with a time resolution of 10ns was designed especially for the purpose of this research (see Fig.4). 
FS-1001Stand with a force gauge FG-5020 by Lutron Co., was used to generate and sense forces applied to the sample.

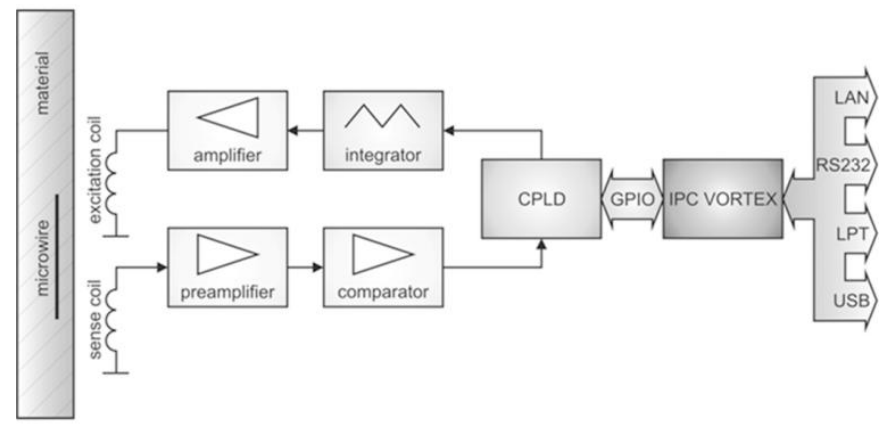

Fig. 4. The block diagram of electronics

\section{EXPERIMENTAL RESULTS}

In all experiments, the frequency of the excitation field $500 \mathrm{~Hz}$ was used and $\mathrm{H}_{\mathrm{M}}$ was set to 600 $\mathrm{A} / \mathrm{m}$. The measured samples (Fig.5.) were composite fibre-glass strips with microwire N38 $\left(\mathrm{Fe}_{38.5} \mathrm{Ni}_{39} \mathrm{Si}_{7.5} \mathrm{~B}_{15}\right)$. Each sample consisted of 20 layers of glass fibre. The microwire was positioned between the $4^{\text {th }}$ and $5^{\text {th }}$ layer. 9 samples were made:

- 1 sample without microwire (Sample 0)

- 2 samples with $1 \mathrm{~cm}$ microwire (Samples 1and 2)

- 2 samples with $2 \mathrm{~cm}$ microwire (Samples 3 and 4)

- 2 samples with $3 \mathrm{~cm}$ microwire (Samples 5 and 6)

- 2 samples with $4 \mathrm{~cm}$ microwire (Samples 7 and 8)

The dimensions of Samples 0-6 were: length: $150 \mathrm{~mm}$, width: $9.5 \mathrm{~mm}$, and thickness: $0.8 \mathrm{~mm}$. Samples 7 and 8 had a length of $200 \mathrm{~mm}$.

The length of the samples was chosen with respect to the length of the embedded microwire and the possibilities of the clamping device. Different lengths of microwires were selected in order to verify the possibilities of the measuring microwire response from the point of view of the designed electronics.

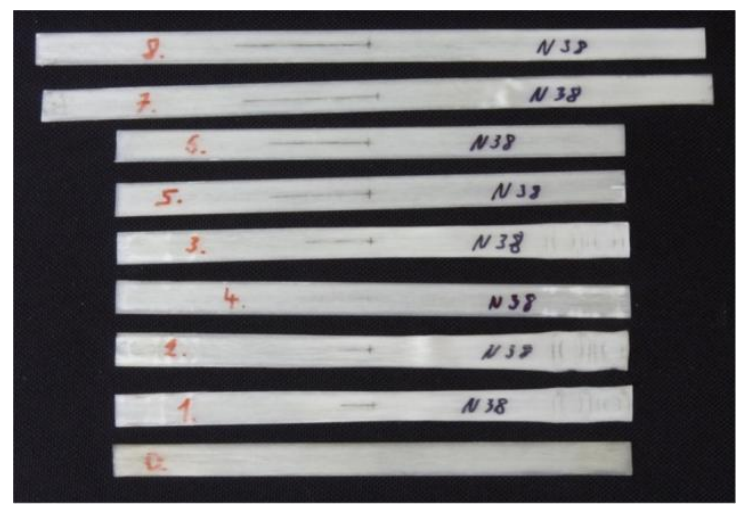

Fig. 5. The block diagram of electronics

For all samples, three types of measurement were performed, with the best results being obtained by using step changed force. Small discrete elongation "stair steps" with increasing and decreasing changes were performed with during 60 seconds for each step. The step of the elongation was $0.05 \mathrm{~mm}$ for a sample (including the elongation of the force gauge). The results are presented in the figures below: 

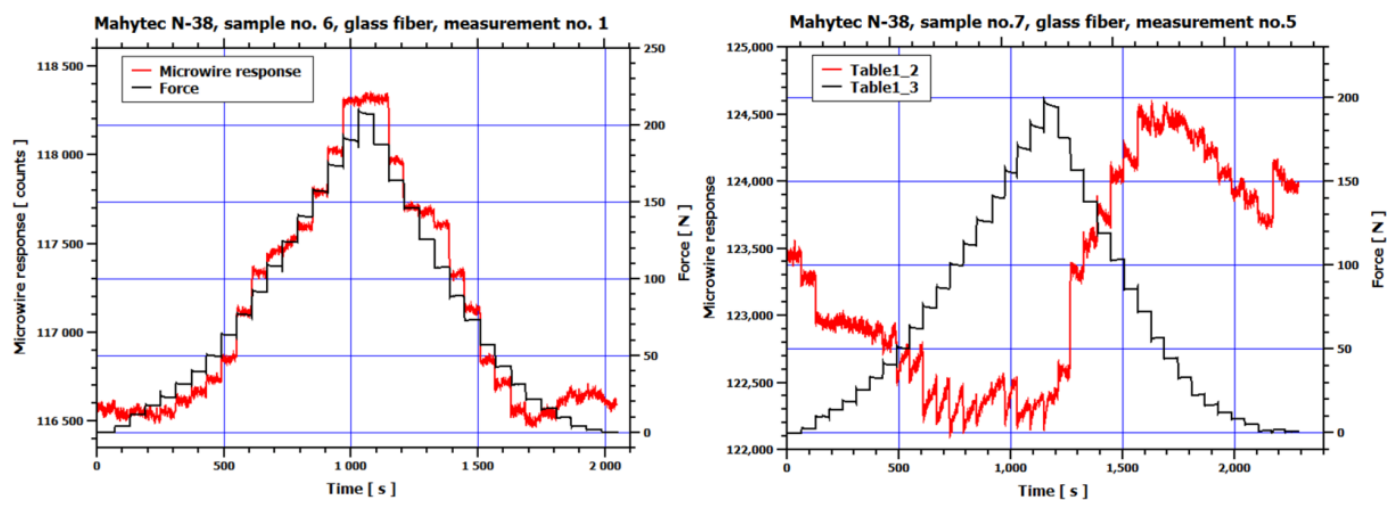

Fig. 6. Step measurement on Sample 6 represents a successfully produced sample on the left and Sample 7 as an unsuccessfully produced one on the right

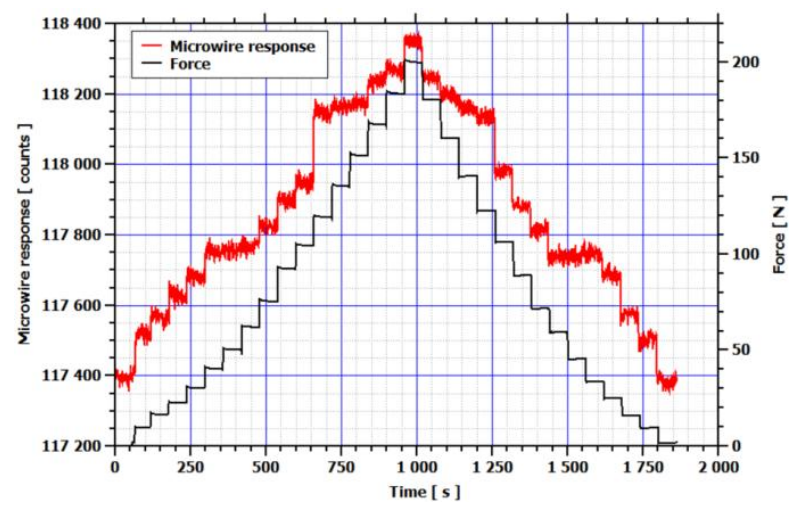

Fig. 7. Step measurement on $S 6$ represents a repeated measurement after approximately six months

Sample 6 reflects tensile stress applied to the sample (see Fig. 6, left). It can be seen that the microwire response returns to the same value as that measured at the beginning of the experiment. In the case of Sample 7, however, (see Fig. 6, right), the response produced a value higher than at the beginning of the measurement. After comparing Samples 6 and 7 we can conclude that some additional stresses were induced in the embedded microwire in Sample 7. With no delay in reaction to the applied stress in either sample, the different reactions to the applied stress can be accounted for by differences in the structural health of the samples. Structural analysis of these samples will be the object of the future research. The measurement presented in Fig. 7 was performed approximately six months after the first measurement on the same sample (Sample6). The different reactions might have been caused by the solidification of the resin used for the production of the samples. This process was observed for all samples.

\section{CONCLUSION}

Possibilities of stress measuring in fibre-glass composite materials were studied. Magnetic microwires were used as sensing devices embedded inside the material. An induction method was used for contactless sensing of microwire parameters and special electronics was developed for the purpose of the experiment. All measurements were performed on the samples prepared as fibreglass strips consisting of 20 layers of glass fibres. Maximum force applied was $200 \mathrm{~N}$, which is approximately equal to $26,315 \mathrm{Mpa}$.

The results obtained with the presented method reveal the possibilities of stress measurement inside of the construction material. This technology is available for almost all non-ferromagnetic materials and can be used for numerous different applications. In comparison with traditional force 
gauges, contactless sensing represents a considerable advantage. The measurements conducted give strong evidence that the response of embedded microwires is dependent on the structural health of the base material.

The exploration of microwires parameters in conjunction with the construction material in which the microwire is embedded allows for in-situ static and dynamic measurements of internal material stresses. Long-term measurements and material status monitoring can be performed, too. As microwires are sensible to the magnetic field, a multisensor for the purposes of navigation and stress monitoring can be prepared.

\section{ACKNOWLADGEMENT}

This work was supported by the Slovak grant APVV-0266-10 under the European grant EDAICET A093 1-RT-GC, SESAMO.

\section{REFERENCES}

[1] V. Zhukova, V. S. Larin, and A. P. Zhukov, J. Appl. Phys. 94, 1115, (2003).

[2] D. Praslicka, J. Blazek, M. Smelko, J. Hudak, I. Mikita, R. Varga, A. Zhukov, In: ICMAST 2012, Abstracts book, p.139, (2012)

[3] R. Varga, K.L. Garcia, A. Zhukov, M. Vazquez, P. Vojtanik, Appl. Phys. Lett. 83 (2003), 2620

[4] E. Komova, M. Varga, R. Varga, P. Vojtanik, J. Bednarcik, J. Kovac, M. Provencio, M. Vazquez, Appl. Phys. Lett.,93, (2008), 062502. 